This is a self-archived - parallel published version of this article in the publication archive of the University of Vaasa. It might differ from the original.

\title{
When Bitcoin has the flu : on Bitcoin's performance to hedge equity risk in the early wake of the COVID-19 outbreak
}

\section{Author(s): Grobys, Klaus}

Title: When Bitcoin has the flu : on Bitcoin's performance to hedge equity risk in the early wake of the COVID-19 outbreak

Year: $\quad 2020$

Version: Accepted manuscript

Copyright (C) 2020 Taylor \& Francis Group. This is an Accepted Manuscript of an article published by Taylor \& Francis in Applied Economics Letters on 26 Jun 2020, available online:

http://www.tandfonline.com/10.1080/13504851.2020.1784380.

\section{Please cite the original version:}

Grobys, K. (2020). When Bitcoin has the flu : on Bitcoin's performance to hedge equity risk in the early wake of the COVID-19 outbreak.

Applied Economics Letters vol.ahead, 1-6.

https://doi.org/10.1080/13504851.2020.1784380 


\title{
When Bitcoin has the flu: On Bitcoin's performance to hedge equity risk in the early wake of the COVID-19 outbreak
}

\author{
Klaus Grobys ${ }^{1}$
}

This draft: June 9, 2020

\begin{abstract}
Using the coronavirus COVID-19 outbreak as a set-up for a quasi-experiment, this study derives novel insights on the dynamic correlation between Bitcoin and U.S. stocks. Given the unprecedented scale of infections and the nature of the virus, the potential impact on the dynamic correlation was unpredictable and therefore uncertain. Using a difference-in-differences setting, the dynamic correlation between Bitcoin and stocks is controlled for the dynamic correlation between gold and stocks. This study finds that Bitcoin performed poorly in hedging this tail risk.
\end{abstract}

JEL classification: G01, G21, G30, G32.

Keywords: Bitcoin, coronavirus, COVID-19, hedging, financial technology

${ }^{1}$ K. Grobys, School of Accounting and Finance, University of Vaasa, Wolffintie 34, 65200 Vaasa, Finland, E-mail:

klaus.grobys@uva.fi.

${ }^{a}$ K. Grobys, Innovation and Entrepreneurship InnoLab, University of Vaasa, Wolffintie 34, 65200 Vaasa, Finland, Email: klaus.grobys@uva.fi. 


\section{Introduction}

Since the World Health Organization (WHO) declared the coronavirus COVID-19 outbreak a public health emergency of international concern on January 30, 2020, it has spread across the globe. On March 11, 2020, WHO Director-General Tedros Adhanom Ghebreyesus characterized COVID-19 as a pandemic. The COVID-19 pandemic has far-reaching consequences beyond the spread of the disease and efforts to quarantine it. The quarantine restriction imposed by the government was followed by one of the most severe stock market crashes in modern times: On March 9, 2020, the U.S. Dow Jones 30 (DJ) index fell 2,013.76 points to 23,851.02. The percentage drop of $7.79 \%$ was one of the worst ever recorded in U.S. history - that is until Thursday, March 12, 2020, where the DJ recorded the largest percentage drop in a day in the DJ's history since Black Monday 1987: The index dropped 2,352.60 points to $21,200.62$-which corresponds to $-9.99 \%$. Unsurprisingly, the U.S. S\&P 500 experienced virtually the same crash.

This paper takes a new perspective and investigates the dynamic correlation between Bitcoin and stocks during the COVID-19 outbreak using the difference-in-differences estimator in a novel quasi-experimental setting. Studying this issue provides important information about the hedging abilities of Bitcoin-which is referred to as 'virtual gold' in the parlance of Dyhrberg (2016) in times when it is needed the most. This study employs the realized dynamic correlation between Bitcoin and the U.S. S\&P500 index as the treatment group, whereas the realized dynamic correlation between gold and the U.S. S\&P500 serves as control group. Furthermore, the sample from April 17, 2015 to October 31, 2019 is used as before-the-event sample, whereas the ex-post pandemic announcement period from March 12, 2020 to March 18, 2020 is the after-the-event sample in this study.

This paper contributes to the literature in some important ways. In a recent paper, Borri (2019) concludes that Bitcoin is exposed to tail-risk within cryptomarkets but it is not exposed to tailrisk with respect to other global assets, like the U.S. equity market or gold. Hence, her results indicate that portfolios of cryptocurrencies could offer attractive returns and hedging abilities when included in an investor's portfolio. In this regard, Shahzad, Bouri, Roubaud, Kristoufek and Lucey (2019) argue that Bitcoin, gold, and the commodity index can be considered as a weak safe-haven asset, whereas Dyhrberg (2016) concludes that Bitcoin can clearly be used as a hedge against stocks. Also, Guesmi, Saadi, Abid and Ftiti (2019) find that using Bitcoin allows hedging the risk investment even for various financial assets. Bouri, Lucey and Roubaud (2020) support Guesmi et al. (2019) and Shahzad et al. (2019) in finding that Bitcoin, Ethereum, and Litecoin are hedges, especially against Asian Pacific and Japanese equities. On the other hand, Bouri, Molnár, Azzi, Roubaud, and Hagfors (2017), who use a dynamic conditional correlation model to explore whether Bitcoin can act as a 
hedge and safe haven for major world stock indices (e.g., bonds, oil, gold, the general commodity index and the US dollar index), show that Bitcoin cannot be regarded as a weak or strong safe haven against extreme movements in any of the assets under study. Finally, Qiang, Bouri, Gupta and Roubaud (2018) use directed acyclic graph as a method to uncover the contemporaneous and lagged relations between Bitcoin and other asset classes. While their findings indicate that the Bitcoin market is quite isolated, the authors find some evidence of lagged relationships between Bitcoin and some assets, in particular in times when Bitcoin faces bear markets. Overall, the literature does not yet agree on a consensus whether cryptocurrencies serve as reliable instruments for hedging equity risks.

In complementing this strand of literature, this is the first study investigating Bitcoin's hedging abilities in the early wake of the COVID-19 outbreak - considered here as an extremely rare event followed by severe declines in stock market prices hardly experienced ever before in financial history. Considering the most recent stock market drops, this is a very serious and important issue. Moreover, this paper steps on new grounds as it employs the recent COVID-19 pandemic as a quasiexperimental design for addressing economic research. As there is no literature on this serious issue yet, this paper remedies this gap in the literature.

\section{Methodology}

Daily price data for Bitcoin and the U.S. S\&P 500 index are downloaded from yahoo.com, whereas daily price data for gold are obtained from nasdaq.com. The data covers a five years period from March 19, 2015 to March 18, 2020. Since Bitcoin is traded 24/7, Bitcoin's sample is matched with the observations for the S\&P 500 and gold leaving us with 1259 daily observations. Log-returns are compounded as

$b t c_{t}=\log \left(\frac{B T C_{t}}{B T C_{t-1}}\right) 100$

$s \& p 500_{t}=\log \left(\frac{S \& P 500_{t}}{s \& P 500_{t-1}}\right) 100$, and

$\operatorname{gold}_{t}=\log \left(\frac{G O L D_{t}}{G O L D_{t-1}}\right) 100$, respectively,

where $B T C_{t}, S \& P 500_{t}, G O L D_{t}$ denote the price data for Bitcoin, the S\&P500, and gold, respectively, given time $t$. Using daily log-returns, we compound the 20-days moving averages of the realized dynamic correlations between Bitcoin and the S\&P500, and between gold and the S\&P500 as 
$\operatorname{corr}(b t c, s \& p 500)_{t}=\frac{\frac{1}{20} \sum_{i=1}^{20}\left(b t c_{t}-\overline{b t}_{t-i}\right)\left(s \& p 500_{t}-\overline{s \& p 500}_{t-i}\right)}{\left.\sqrt{\frac{1}{20} \sum_{i=1}^{20}\left(b t c_{t}-\overline{b t c} t-i\right)} \sqrt{\frac{1}{20} \sum_{i=1}^{20}\left(s \& p 500_{t}-\overline{s \& p 500} t-i\right.}\right)}$, and

$\operatorname{corr}\left(\text { gold }_{,} s \& 500\right)_{t}=\frac{\frac{1}{20} \sum_{i=1}^{20}\left(\text { gold }_{t}-\overline{\operatorname{gold}}_{t-i}\right)\left(s \& p 5 \quad t^{-} \overline{s \& p 500}_{t-i}\right)}{\sqrt{\frac{1}{20} \sum_{i=1}^{20}\left(\operatorname{gold}_{-} \overline{\operatorname{gold}}_{t-i}\right)} \sqrt{\frac{1}{20} \sum_{i=1}^{20}\left(s \& p 500_{t}-\overline{s \& p 500}_{t-i}\right)}}$

where $\overline{b t c}_{t-i}=\frac{1}{20} \sum_{i=1}^{20} b t c_{t-i},{\overline{s \& p 500_{t-i}}}_{10}=\frac{1}{20} \sum_{i=1}^{20} s \& p 500_{t-i}$, and $\overline{\operatorname{gold}}_{t-i}=\frac{1}{20} \sum_{i=1}^{20}$ gold $_{t-i}$. The realized dynamic correlations are plotted in figure 1. Having more than 1000 in-sample observations, according to the Law of Large Numbers the sample means for $\operatorname{corr}(b t c, s \& p 500)_{t}$ and $\operatorname{corr}(\text { gold, } s \& p 500)_{t}$ converge to their true expectations.

In line with Chinese government data reviewed by South China Morning Post, the first case of the novel coronavirus emerged on November 17, 2019. ${ }^{1}$ Therefore, we use the sample from April 17, 2015 to October 31, 2019 as before-the-event sample (e.g., sample 1). ${ }^{2}$ As WHO Director Dr. Tedros Adhanom characterized COVID-19 as a pandemic on 11 March, 2020, the sample from March 12, 2020 to March 18, 2020 is this study's after-the-event sample (e.g., sample 2). In our difference-in-difference estimation, we use $\operatorname{corr}(b t c, s \& p 500)_{t}$ as the treatment group and $\operatorname{corr}(\text { gold, } s \& p 500)_{t}$ as the control group. The rationale to use the dynamic correlation between gold and U.S. stocks as a control group is based on the literature documenting that gold serves as a hedge against stocks on average and a safe haven in extreme stock market conditions (Baur and Lucey, 2010; Baur and McDermott, 2010). In table 1 we report the distribution of the realized dynamic correlations, whereas table 2 reports the difference-in-differences estimation results.

From table 1 we learn that that the distributions of $\operatorname{corr}(\text { gold, } s \& p 500)_{t}$ and $\operatorname{corr}(b t c, s \& p 500)_{t}$ have similar properties. However, $\operatorname{corr}(\text { gold, } s \& p 500)_{t}$ tends to exhibit higher negative realized dynamic correlations, because $2.50 \%$ of the distribution exhibit values lower than 0.6629 , whereas the corresponding figure for $\operatorname{corr}(b t c, s \& p 500)_{t}$ is -0.5017 . Interestingly, the correlation between $\operatorname{corr}(\text { gold, } s \& p 500)_{t}$ and $\operatorname{corr}(b t c, s \& p 500)_{t}$ is -0.1295 in the April 17, 2015 to October 31, 2019 period. Moreover, using

$t_{g o l d, s \& p 500}=\frac{\overline{\operatorname{corr}(\text { gold }, s \& p 50)_{t}}}{\sqrt{\left(\frac{1-\left(\operatorname{corr}(\text { gold,s\&p500)t })^{2}\right.}{N-2}\right)}}$, and $t_{b t c, s \& p 500}=\frac{\overline{\operatorname{corr}(b t c, s \& p 500)_{t}}}{\sqrt{\left(\frac{1-\left(\operatorname{corr}(b t c, s \& p 500)_{t}\right)^{2}}{N-2}\right)}}$

\footnotetext{
${ }^{1}$ See https://www.theguardian.com/world/2020/mar/13/first-covid-19-case-happened-in-november-china-governmentrecords-show-report.

${ }^{2}$ As the virus could have emerged even earlier, skipping the 17 days period between October 31, 2019 and November 17, 2019 controls for any potential microstructure issues.
} 
to estimate the statistical significance of the sample means of the realized dynamic correlations gives $t$-statistics of -2.63 and -0.70 , indicating that only gold is statistically significantly negatively correlated with the S\&P500 over the sample period from April 17, 2015 to October 31, 2019. Next, from table 2 we observe that the sample means for $\operatorname{corr}(\text { gold, } s \& p 500)_{t}$ and $\operatorname{corr}(b t c, s \& p 500)_{t}$ in the ex-post pandemic announcement period are -0.0633 and 0.6353 , respectively. ${ }^{3}$ The average realized correlation corresponding to -0.0633 is clearly within the $95 \%$ interval of the empirical probability distribution for $\operatorname{corr}(\text { gold, } s \& p 500)_{t}$. This result confirms the earlier literature suggesting that gold is a safe haven as the correlation between gold and U.S. stocks is in the early wake of the COVID-19 outbreak statistically not different from the before-the-event time sample average. Having in mind the current literature on Bitcoin it is surprising to observe that the average realized correlation between Bitcoin and U.S. stocks corresponding to 0.6353 is far outside of the $99 \%$ interval of the empirical probability distribution for $\operatorname{corr}(b t c, s \& p 500)_{t}$. This suggests that the sample average of $\operatorname{corr}(b t c, s \& p 500)_{t}$ is statistically significantly higher in the ex-post pandemic announcement period.

The question arises, whether the difference of $\operatorname{corr}(b t c, s \& p 500)_{t}$ is significant even after accounting for the control group. To address this issue, we calculate the difference-ofdifferences between the treatment group, $\operatorname{corr}(b t c, s \& p 500)_{t}$, and the control group, $\operatorname{corr}(\operatorname{gold}, s \& p 500)_{t}$. From table 2 it is evident that the point estimate corresponding to 0.6418 is statistically significant on any level. ${ }^{4}$ To check the robustness of the results, the realized dynamic correlations are re-estimated using Equations (1) - (2) but with rolling time-windows of either 15- or 25-days instead of 20-days. The results are reported in tables A.1.-A.4. in the appendix. The results are virtually the same.

While this result is in stark contrast to the results documented in Borri (2019), Bouri et al. (2020), and Shahzad et al. (2019), it supports Bouri et al. (2017) who conclude that Bitcoin cannot be regarded as a weak or strong safe haven against extreme movements in bonds, oil, gold, the general commodity index and the US dollar index. Specifically, the result of the current study suggests that Bitcoin cannot be regarded as a weak (or strong) safe haven against extreme movements in U.S. equities either.

\footnotetext{
${ }^{3}$ The realized dynamic correlations for $\operatorname{corr}(g o l d, s \& p 500)_{t}$ and $\operatorname{corr}(b t c, s \& p 500)_{t}$ vary between -0.0059 and 0.2626 , and 0.6196 to 0.6587 in the March 12, 2020 to March 18, 2020 period.

4 The estimated difference of 0.6275 corresponds in both realized dynamic correlation distributions, that is, $\operatorname{corr}(b t c, s \& p 500)_{t}$ or $\operatorname{corr}(\text { gold, } s \& p 500)_{t}$ to less than $0.5 \%$ of the probability mass. Note that Rehse, Riordan, Rottke, ans Zietz (2019), who explore the effects of the hurricane Sandy on market liquidity, also employ a differencein-differences setting using the outbreak of the hurricane as quasi-experimental design.
} 
As an additional robustness check, the period from January 31, 2020 until March 18, 2020 is chosen as after-the-event sample because the WHO declared the outbreak a Public Health Emergency of International Concern (PHEIC) already on January 30, 2020. The sample period January 31, 2020 until March 18, 2020 corresponds to 33 trading days. From table 3 we observe that in the after-the-event sample neither the sample average of the dynamic correlation between Bitcoin and U.S. stocks nor sample average of the dynamic correlation between gold and U.S. stocks is statistically different from the corresponding before-the-event sample averages. However, we observe that the difference-in-differences corresponding to a point estimate of 0.4923 is statistically significant on a 5\%. This result shows that even if an extended after-the-event sample is employed, Bitcoin performed poorly in hedging this tail risk in the U.S. stock market.

\section{Conclusion}

This paper employs the outbreak of the pandemic as a quasi-experimental design to explore the hedging abilities of Bitcoin - which has been referred to as 'virtual gold' in the earlier literature to highlight it's renowned abilities to hedge risk investment for various financial assets classes, especially stocks. Using a difference-in-differences setting, the realized dynamic correlation between gold and U.S. stocks serves as control group, whereas the realized dynamic correlation between Bitcoin and U.S. stocks is this study's treatment group. The findings suggest that Bitcoin does not serve as a useful tool for hedging this extraordinary tail risk in U.S. stocks. This implies limited hedging abilities of Bitcoin - especially in times of severe economic stress - and the need to follow a dynamic approach when hedging equity market risk with Bitcoin. Future research is strongly encouraged to study the economic impact of the coronavirus COVID-19 outbreak in other economic research settings. For instance, so far more than 100 countries have travel restrictions imposed by the governments. Moreover, governments' impositions to stay at home might have a devastating impact on the hotel and restaurant industry. Investigating the impact of the COVID-19 pandemic on industry sectors is encouraged for future research. Furthermore, the decline in Bitcoin's price on March 12th could be also affected by margin trading on the cryptocurrency exchange Bitmex. For instance, when Bitcoin's price fell, a considerable amount of positions were liquidated causing a further decline. Hence, the decrease in Bitcoin's price was not exclusively due to the COVID-19 pandemic but also a consequence of some market microstructure issues associated with cryptocurrency exchanges. Future research is encouraged to explore this issue in more detail. 


\section{References}

Baur D. G., Lucey B. M., 2010. Is gold a hedge or a safe haven? An analysis of stocks, bonds, and gold, Financial Review 45, 217-229.

Baur D. G., McDermott, T.K., 2010. Is gold a safe haven? International evidence, Journal of Banking and Finance 34, 1886-1898.

Borri, N., 2019. Conditional tail-risk in cryptocurrency markets, Journal of Empirical Finance 50, 119.

Bouri, E., Molnár, P., Azzi, G., Roubaud, D., Hagfors, L.I., 2017. On the hedge and safe haven properties of Bitcoin: Is it really more than a diversifier? Finance Research Letters 20, $192-198$.

Bouri, E., Lucey, B., Roubaud, D., 2020. Cryptocurrencies and the downside risk in equity investments, Finance Research Letters 33, 101211.

Dyhrberg, A., 2016. Hedging capabilities of bitcoin. Is it the virtual gold? Finance Research Letters $16,139-144$.

Guesmi, K., Saadi, S., Abid, I., and Ftiti, Z., 2019. Portfolio diversification with virtual currency: Evidence from bitcoin, International Review of Financial Analysis 63, 431-437.

Qiang, J., Bouri, E., Gupta, R., Roubaud, D., 2018. Network causality structures among Bitcoin and other financial assets: A directed acyclic graph approach, The Quarterly Review of Economics and Finance 70, $203-213$.

Rehse, D., Riordan, R., Rottke, N., and Zietz, J., 2019. The effects of uncertainty on market liquidity: Evidence from Hurricane Sandy, Journal of Financial Economics 134, 318-332.

Shahzad, S.J.H., Bouri, E., Roubaud, D., Kristoufek, L. and Lucey, B., 2019. Is Bitcoin a better safehaven investment than gold and commodities? International Review of Financial Analysis 63, 322-330. 


\section{Figures and tables}

\section{Figure 1. Realized dynamic correlations}

This figure plots the realized dynamic correlations between Bitcoin and the S\&P500 and gold and the S\&P500 across time. The sample is from April 2015 to March 2020.

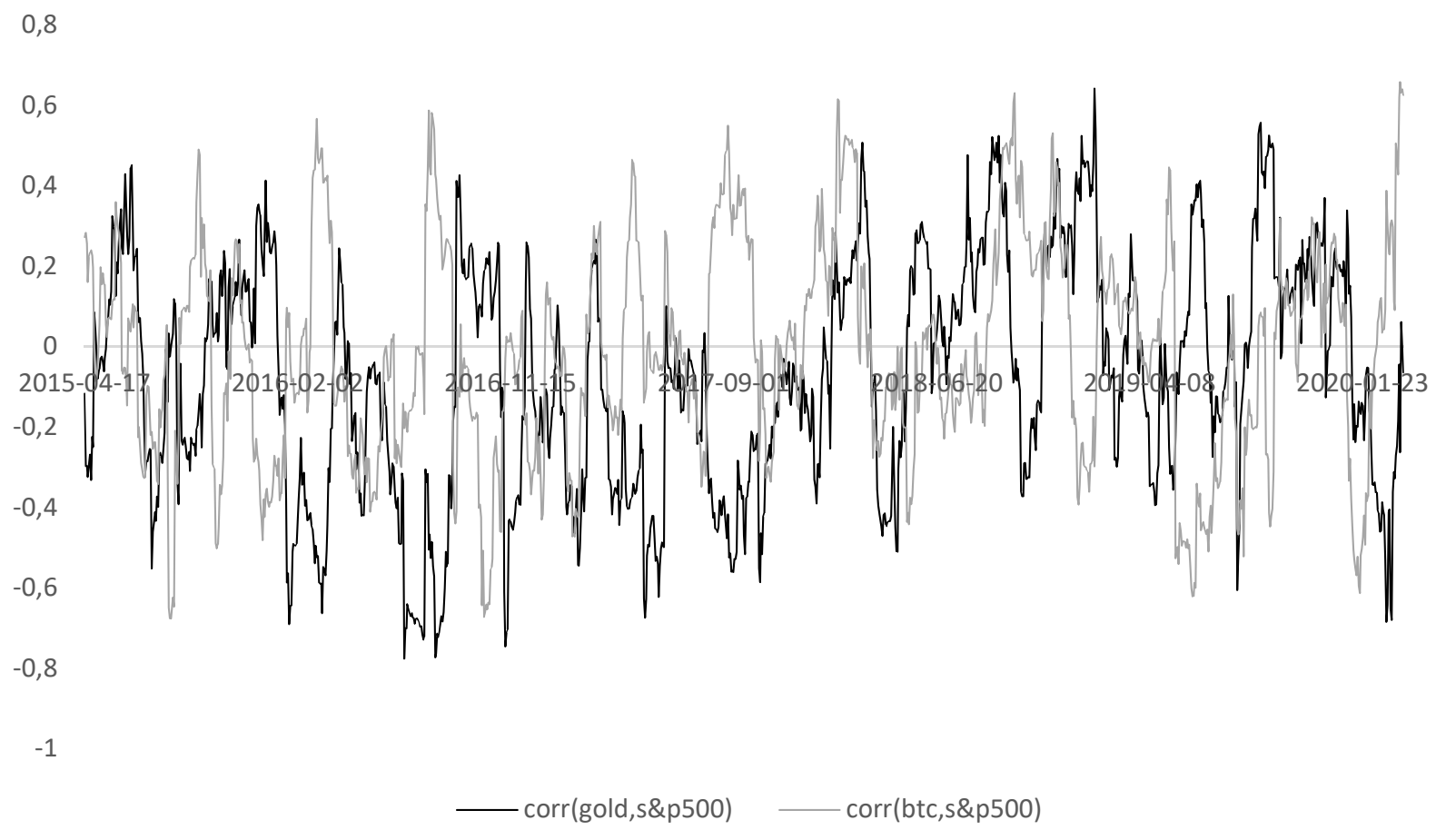




\section{Table 1. Distributions of realized dynamic correlations}

This table reports the distribution of the realized dynamic correlations between gold and the S\&P500 and between Bitcoin and the S\&P500. The sample period is from April 17, 2015 to October 31, 2019.

\begin{tabular}{ccc}
\hline Probability $<$ & $\boldsymbol{c o r r}(\boldsymbol{g o l d}, \boldsymbol{s} \& \boldsymbol{p 5 0 0})_{t}$ & $\boldsymbol{c o r r}(\boldsymbol{b t c}, \boldsymbol{s} \& \boldsymbol{p 5 0 0})_{t}$ \\
\hline $\mathbf{0 . 0 5} \%$ & -0.7212 & -0.6471 \\
$\mathbf{2 . 5 \%}$ & -0.6629 & -0.5017 \\
$\mathbf{5 \%}$ & -0.5476 & -0.4431 \\
$\mathbf{1 0 \%}$ & -0.4539 & -0.3611 \\
$\mathbf{2 5 \%}$ & -0.3048 & -0.2008 \\
$\mathbf{M e d i a n}$ & -0.0720 & -0.0263 \\
$\mathbf{7 5 \%}$ & 0.1600 & 0.1630 \\
$\mathbf{9 0 \%}$ & 0.2961 & 0.3383 \\
$\mathbf{9 5 \%}$ & 0.4019 & 0.4461 \\
$\mathbf{9 7 . 5 \%}$ & 0.4590 & 0.4948 \\
$\mathbf{9 9 . 5 \%}$ & 0.5255 & 0.5814 \\
\hline
\end{tabular}




\section{Table 2. Difference-in-Differences estimation}

This table reports the sample averages for the realized dynamic correlations between Bitcoin and the S\&P500 and between gold and the S\&P500. The realized dynamic correlations are calculated using a rolling-time window of 20-days. We use the sample from April 17, 2015 to October 31, 2019 as before-the-event sample (e.g., sample 1), whereas the sample from March 12, 2020 to March 18, 2020 is the after-the-event sample (e.g., sample 2). The reported $p$-values are based on the empirical distribution of the dynamic correlations $\operatorname{corr}(b t c, s \& p 500)_{t}$, and $\operatorname{corr}(g o l d, s \& p 500)_{t}$, respectively, whereas the $t$-statistics are calculated as in Equation (3).

\begin{tabular}{lccc}
\hline Sample & $\boldsymbol{c o r r}(\boldsymbol{b t c}, \boldsymbol{s} \& \boldsymbol{p 5 0 0})_{\boldsymbol{t}}$ & $\boldsymbol{c o r r}(\boldsymbol{g o l d}, \boldsymbol{s} \& \boldsymbol{p 5 0 0})_{\boldsymbol{t}}$ & Difference \\
\hline Sample 1 & -0.0208 & $-0.0776^{* * *}$ & \\
$(t$-statistic $)$ & $(-0.70)$ & $(-2.63)$ & \\
& & & \\
Sample 2 & $0.6353^{* * *}$ & -0.0633 & \\
$(p$-value $)$ & $(0.0000)$ & $(0.5179)$ & \\
& & & $0.6418^{* * *}$ \\
Difference & $0.6561^{* * *}$ & 0.0143 & $(0.000)$ \\
$(p$-value $)$ & $(0.0000)$ & $(0.3913)$ & \\
\hline
\end{tabular}

*** Statistical significance on a 1\% level. 


\section{Table 3. Difference-in-Differences estimation}

This table reports the sample averages for the realized dynamic correlations between Bitcoin and the S\&P500 and between gold and the S\&P500. The realized dynamic correlations are calculated using a rolling-time window of 20-days. We use the sample from April 17, 2015 to October 31, 2019 as before-the-event sample (e.g., sample 1), whereas the sample from January 31, 2020 to March 18, 2020 is the after-the-event sample (e.g., sample 2). The reported $p$-values are based on the empirical distribution of the dynamic correlations $\operatorname{corr}(b t c, s \& p 500)_{t}$, and $\operatorname{corr}(g o l d, s \& p 500)_{t}$, respectively, whereas the $t$-statistics are calculated as in Equation (3).

\begin{tabular}{lccc}
\hline Sample & $\boldsymbol{c o r r}(\boldsymbol{b t c}, \boldsymbol{s} \& \boldsymbol{p 5 0 0})_{\boldsymbol{t}}$ & $\boldsymbol{c o r r}(\boldsymbol{g o l d}, \boldsymbol{s} \& \boldsymbol{p 5 0 0})_{\boldsymbol{t}}$ & Difference \\
\hline Sample 1 & -0.0208 & $-0.0776^{* * *}$ & \\
$(t$-statistic $)$ & $(-0.70)$ & $(-2.63)$ & \\
& & & \\
Sample 2 & 0.2063 & -0.3428 & \\
$(p$-value $)$ & $(0.2114)$ & $(0.2105)$ & \\
& & & $0.4923^{* *}$ \\
Difference & 0.2271 & -0.2652 & $(0.0271)$ \\
$(p$-value $)$ & $(0.1956)$ & $(0.2891)$ & \\
\hline
\end{tabular}

** Statistical significance on a 5\% level. 


\section{Appendix}

Table A.1. Distributions of realized dynamic correlation using dynamic correlations based on

\section{5-days rolling time-windows}

This table reports the distribution of the realized dynamic correlations between gold and the S\&P500 and between Bitcoin and the S\&P500. The realized dynamic correlations are calculated using rolling-time windows of 15-days. The sample period is from April 17, 2015 to October 31, 2019.

\begin{tabular}{ccc}
\hline Probability $<$ & $\boldsymbol{c o r r}(\boldsymbol{g o l d}, \boldsymbol{s} \& \boldsymbol{p 5 0 0})_{\boldsymbol{t}}$ & $\boldsymbol{c o r r}(\boldsymbol{b t c}, \boldsymbol{s} \& \boldsymbol{p 5 0 0})_{\boldsymbol{t}}$ \\
\hline $\mathbf{0 . 0 5 \%}$ & -0.7496 & -0.6804 \\
$\mathbf{2 . 5 \%}$ & -0.6808 & -0.5771 \\
$\mathbf{5 \%}$ & -0.6258 & -0.4961 \\
$\mathbf{1 0 \%}$ & -0.5070 & -0.4240 \\
$\mathbf{2 5 \%}$ & -0.2756 & -0.3251 \\
$\mathbf{M e d i a n}$ & -0.0842 & -0.0232 \\
$\mathbf{7 5 \%}$ & 0.1672 & 0.1931 \\
$\mathbf{9 0 \%}$ & 0.3448 & 0.4120 \\
$\mathbf{9 5 \%}$ & 0.4414 & 0.4756 \\
$\mathbf{9 7 . 5 \%}$ & 0.5035 & 0.5441 \\
$\mathbf{9 9 . 5 \%}$ & 0.5937 & 0.6790 \\
\hline
\end{tabular}


Table A.2 Difference-in-Differences estimation using dynamic correlations based on 15-days rolling time-windows

This table reports the sample averages for the realized dynamic correlations between Bitcoin and the S\&P500 and between gold and the S\&P500. The realized dynamic correlations are calculated using rolling-time windows of 15 -days. We use the sample from April 17, 2015 to October 31, 2019 as before-the-event sample (e.g., sample 1), whereas the sample from March 12, 2020 to March 18, 2020 is the after-the-event sample (e.g., sample 2). The reported $p$-values are based on the empirical distribution of the dynamic correlations $\operatorname{corr}(b t c, s \& p 500)_{t}$, and $\operatorname{corr}(\text { gold, } s \& p 500)_{t}$, respectively, whereas the $t$-statistics are calculated as in Equation (3).

\begin{tabular}{lccc}
\hline Sample & $\boldsymbol{c o r r}(\boldsymbol{b t c}, \boldsymbol{s} \& \boldsymbol{p 5 0 0})_{t}$ & $\boldsymbol{c o r r}(\boldsymbol{g o l d}, \boldsymbol{s} \& \boldsymbol{p 5 0 0})_{t}$ & Difference \\
\hline Sample 1 & -0.0234 & $-0.0760^{* * *}$ & \\
$(t$-statistic $)$ & $(-0.7910)$ & $(-2.5770)$ & \\
& & & \\
Sample 2 & $0.6439 * *$ & -0.0725 & \\
$(p$-value $)$ & $(0.0114)$ & $(0.4996)$ & \\
& & & $0.6638^{* * *}$ \\
Difference & $0.6673^{* * *}$ & 0.0035 & $(0.0087)$ \\
$(p$-value $)$ & $(0.0079)$ & $(0.4192)$ &
\end{tabular}

*** Statistical significance on a $1 \%$ level.

** Statistical significance on a $5 \%$ level. 
Table A.3. Distributions of realized dynamic correlation using dynamic correlations based on 25-days rolling time-windows

This table reports the distribution of the realized dynamic correlations between gold and the S\&P500 and between Bitcoin and the S\&P500. The realized dynamic correlations are calculated using rolling-time windows of 25-days. The sample period is from April 17, 2015 to October 31, 2019.

\begin{tabular}{ccc}
\hline Probability $<$ & corr $(\text { gold, } \boldsymbol{s} \& \boldsymbol{p 5 0 0})_{t}$ & $\boldsymbol{c o r r}(\boldsymbol{b t c}, \boldsymbol{s} \& \boldsymbol{p 5 0 0})_{\boldsymbol{t}}$ \\
\hline $\mathbf{0 . 0 5 \%}$ & -0.7079 & -0.6174 \\
$\mathbf{2 . 5 \%}$ & -0.6339 & -0.4589 \\
$\mathbf{5 \%}$ & -0.5109 & -0.3797 \\
$\mathbf{1 0} \%$ & -0.4239 & -0.3126 \\
$\mathbf{2 5 \%}$ & -0.2906 & -0.1701 \\
$\mathbf{M e d i a n}$ & -0.0815 & -0.0281 \\
$\mathbf{7 5 \%}$ & 0.1362 & 0.1411 \\
$\mathbf{9 0 \%}$ & 0.2700 & 0.3027 \\
$\mathbf{9 5 \%}$ & 0.3496 & 0.4000 \\
$\mathbf{9 7 . 5 \%}$ & 0.4001 & 0.4498 \\
$\mathbf{9 9 . 5 \%}$ & 0.4478 & 0.5016 \\
\hline
\end{tabular}


Table A.2 Difference-in-Differences estimation using dynamic correlations based on 25-days rolling time-windows

This table reports the sample averages for the realized dynamic correlations between Bitcoin and the S\&P500 and between gold and the S\&P500. The realized dynamic correlations are calculated using rolling-time windows of 25-days. We use the sample from April 17, 2015 to October 31, 2019 as before-the-event sample (e.g., sample 1), whereas the sample from March 12, 2020 to March 18, 2020 is the after-the-event sample (e.g., sample 2). The reported $p$-values are based on the empirical distribution of the dynamic correlations $\operatorname{corr}(b t c, s \& p 500)_{t}$, and $\operatorname{corr}(\text { gold, } s \& p 500)_{t}$, respectively, whereas the $t$-statistics are calculated as in Equation (3).

\begin{tabular}{lccc}
\hline Sample & $\boldsymbol{c o r r}(\boldsymbol{b t c}, \boldsymbol{s} \& \boldsymbol{p 5 0 0})_{t}$ & $\boldsymbol{c o r r}(\boldsymbol{g o l d}, \boldsymbol{s} \& \boldsymbol{p 5 0 0})_{t}$ & Difference \\
\hline Sample 1 & -0.0170 & $-0.0795^{* * *}$ & \\
$(t$-statistic $)$ & $(-0.5740)$ & $(-2.6900)$ & \\
& & & \\
Sample 2 & $0.6426^{* * *}$ & -0.0583 & \\
$(p$-value $)$ & $(0.0000)$ & $(0.4996)$ & \\
& & & $0.6384^{* * *}$ \\
Difference & $0.6596^{* * *}$ & 0.0212 & $(0.0000)$ \\
$(p$-value $)$ & $(0.0000)$ & $(0.4649)$ & \\
\hline
\end{tabular}

*** Statistical significance on a $1 \%$ level. 